\title{
Reno-protection of Urine-derived Stem Cells in A Chronic Kidney Disease Rat Model Induced by Renal Ischemia and Nephrotoxicity
}

\author{
Chao Zhang1,2, Sunil K. George1, Rongpei Wu1,3, Parth Udayan Thakker4, Mehran Abolbashari1, \\ Tae-Hyoung Kim ${ }^{1,5}$, In Kap Ko ${ }^{1}$, Yuanyuan Zhang ${ }^{1 凶}$, Yinghao Sun², John Jackson¹, Sang Jin Lee ${ }^{1}$, James J. \\ $\mathrm{Yoo}^{1}$, and Anthony Atala ${ }^{1}$ \\ 1. Wake Forest Institute for Regenerative Medicine, Wake Forest School of Medicine, Medical Center Boulevard, Winston-Salem, NC, USA \\ 2. Department of Urology, Changhai Hospital, the Second Military Medical University, 168 Changhai Road, Shanghai, People's Republic of China \\ 3. Department of Urology, First Affiliated Hospital of Sun Yat-Sen University, Guangzhou, Guang Dong, People's Republic of China. \\ 4. Department of Urology, Wake Forest Baptist Medical Center, Medical Center Boulevard, Winston-Salem, NC, USA \\ 5. Department of Urology, College of Medicine, Chung-Ang University, Seoul, South Korea \\ $\bowtie$ Corresponding author: Yuanyuan Zhang, yzhang@wakehealth.edu Tel: +13367131189; Fax: 13367137990
}

(0) The author(s). This is an open access article distributed under the terms of the Creative Commons Attribution License (https://creativecommons.org/licenses/by/4.0/). See http://ivyspring.com/terms for full terms and conditions.

Received: 2019.06.12; Accepted: 2019.09.30; Published: 2020.01.01

\begin{abstract}
Purpose: Drug-induced nephrotoxicity can occur in patients with pre-existing renal dysfunction or renal ischemia, potentially leading to chronic kidney disease (CKD) and end-stage renal disease (ESRD). Prompt treatment of CKD and the related side effects is critical in preventing progression to ESRD. The goal of this study was to demonstrate the therapeutic potential of urine-derived stem cells (USC) to treat chronic kidney disease-induced by nephrotoxic drugs and renal ischemia.

Materials and methods: Human USC were collected, expanded and characterized by flow cytometry. A CKD model was induced by creating an ischemia-reperfusion injury and gentamicin administration. Twenty-eight adult immunodeficient rats were divided into three groups: PBS-treated group $(n=9)$, USC-treated group $(n=9)$, and sham group with age-matched control animals $(n=10)$. Cell suspension of USC $\left(5 \times 10^{6} / 100 \mu l /\right.$ kidney $)$ or PBS was injected bilaterally into the renal parenchyma 9 weeks after CKD model creation. Renal function was evaluated by collection blood and urine samples to measure serum creatinine and glomerulus filtration rate. The kidneys were harvested 12 weeks after cell injection. Histologically, the extent of glomerulosclerosis and tubular atrophy, the amount of collagen deposition, interstitial fibrosis, inflammatory monocyte infiltration, and expression of transforming growth factor beta 1 (TGF-B1), and superoxide dismutase 1 (SOD-1) were examined.

Results: USC expressed renal parietal epithelial cells (CD24, CD29 and CD44). Renal function, measured by GFR and serum $\mathrm{Cr}$ in USC-treated group were significantly improved compared to PBS-treated animals $(p<0.05)$. The degree of glomerular sclerosis and atrophic renal tubules, the amount of fibrosis, and monocyte infiltration significantly decreased in USC-treated group compared to the PBS group $(p<0.05)$. The level of TGF- $B 1$ expression in renal tissues was also significantly lower in the PBS group, while the level of SOD-1 expression was significantly elevated in the USC group, compared to PBS group $(p<0.05)$.

Conclusions: The present study demonstrates the nephron-protective effect of USC on renal function via anti-inflammatory, anti-oxidative stress, and anti-fibrotic activity in a dual-injury CKD rat model. This provides an alternative treatment for CKD in certain clinical situations, such as instances where CKD is due to drug-induced nephrotoxicity and renal ischemia.
\end{abstract}

Key words: Urine-derived Stem Cell, Chronic Kidney Disease, Kidney degeneration 


\section{Introduction}

Chronic kidney disease (CKD) is a major health problem characterized by a gradual loss of kidney function over time. Over 30 million people or $15 \%$ of US adults are estimated to have CKD ${ }^{1}$. CKD may progress to end-stage renal disease (ESRD), which is a public health concern and big economic burden. ESRD costs the United States taxpayers approximately 32.8 billion dollars in annual Medicare expenditures [2]. Causes of CKD vary widely, however drug-induced nephrotoxicity has been an increasingly recognized complication of many therapeutic agents in the clinical setting. In patients with pre-existing renal dysfunction or renal ischemia, the effects of drug-induced nephrotoxicity can be profound and may accelerate progression to ESRD ${ }^{2}$. At present, hemodialysis and renal transplantation are both effective treatment modalities for ESRD. However, hemodialysis is expensive with many potential complications while renal transplant as a treatment modality is limited by the number organ donors available ${ }^{3}$.

Cell therapy has emerged as a promising therapeutic approach for the treatment of CKD with great potential ${ }^{4}$. Several stem cell types from a variety of sources have been used in animal experiments ${ }^{5-10}$. Bone marrow-derived stem cells were found to significantly improve renal function in mice models 5 , and their safety was subsequently studied in a phase 1 clinical trial ${ }^{11}$. In addition, stem cells derived from amniotic fluid ${ }^{6,7}$, endothelium ${ }^{8}$, adipose tissue ${ }^{9}$ and primary renal cells ${ }^{10}$ have demonstrated therapeutic effect in renal regeneration. However, obtaining these stem cells requires invasive procedures, which may cause iatrogenic injury to adjacent organs and increases infection risk among other complications. Thus, the desire to obtain stem cells from a simple, non-invasive and inexpensive route is of utmost necessity.

Recently, we demonstrated that stem cells exist in human urine and possess the potential for clinical applications ${ }^{12-20}$. Urine derived stem cells (USC) are thought to originate from the parietal cells in renal glomeruli 14 and have the potential for tissue regenerative effects, including robust proliferative potential, multi-potential differentiation, and the ability to exert regenerative effects via paracrine factors $14,21,22$. USC secrete multiple paracrine factors to recruit native cells to participate in tissue regeneration and induce immune-modulatory changes in vivo. Studies from our group and other laboratories have demonstrated that USC or the media derived from USC significantly improved renal function by reducing inflammation and fibrin matrix deposition within the kidney in different rodent CKD models, including age-related kidney disease 23 and streptozotocin-induced diabetic nephropathy 24,25. Furthermore, USC enhanced renal function in an acute renal ischemia model ${ }^{26}$. Nephrotoxic drug induced renal damage superimposed on pre-existing renal damage is one of the more common causes of iatrogenic CKD ${ }^{2}$. However, it is challenge to revise the renal function and prevent histological structure damages from the chronic renal damages in clinical settings. Compared to stem cells derived from other sources such as amniotic fluid, placenta, bone marrow, adipose tissues, USC have unique advantages in the treatment of CKD. USC can be obtained from simple, safe, non-invasive and low-cost approaches. A large of amount of stem cells can be generated through a few weeks. In addition, as the kidney tissue specific stem cells, USC might be optimal for the kidney tissue repair. As such, the goal of this study was to determine therapeutic impact of USC on renal parenchyma architecture and renal function in a rat CKD model, induced by dual nephrotoxic drug-renal ischemia injury.

\section{Methods}

\section{USC isolation and identification}

Collection of human urine samples for USC isolation in this study was approved by the Wake Forest University Health Sciences Institutional Review Board. USC culture and characterization have been described previously ${ }^{12-20}$. In brief, USC were collected from healthy adult males $(n=6)$ and expanded in culture media comprised of keratinocyte serum-free medium (Gibco, Gaithersburg, MD) and Dulbecco's Modified Eagle's medium (DMEM, high glucose, Gibco, Gaithersburg, MD) with 5\% fetal bovine serum (FBS) (Gibco, Gaithersburg, MD) containing supplements ${ }^{12}$, see Table 1. Expanded USC were trypsinized and incubated with antihuman antibody labeled with cell surface markers of renal parietal epithelial cells (CD24, CD29 and CD44) (BD, Franklin Lakes, NJ) ${ }^{27}$, mesenchymal stem cells (MSC) (CD73, CD90, CD105, and CD146) (BD, Franklin Lakes, NJ), embryonic stem cells (SSEA4), hematopoietic stem cell (CD31, CD34, CD45) (BD, Franklin Lakes, NJ) and STRO-1 (BioLegend, San Diego, CA). Fluorescein isothiocyanate (FITC) or phycoerythrin (PE) conjugated isotype antibodies were used to determine background fluorescence. All cells were analyzed using BD FACS Caliber analytical fluorescence activated cell sorter (BD, Franklin Lakes, $\mathrm{NJ})$. 
Table 1. Antibodies, mediums, and serums used in this study.

\begin{tabular}{lllll}
\hline Items & Antibody & Type & Source & Catalog No. \\
\hline List of & CD24 & FITC-conjugate & BD & 560992 \\
Antibodies & CD29 & PE-conjugated & BD & 555443 \\
& CD31 & FITC-conjugated & BD & 560984 \\
& CD34 & APC-conjugated & BD & 560940 \\
& CD44 & PE-conjugated & BD & 550989 \\
& CD45 & APC-conjugated & BD & 340943 \\
& CD73 & PE-conjugated & BD & 550257 \\
& CD90 & APC-conjugated & BD & 561971 \\
& CD105 & PE-conjugated & BD & 560839 \\
& CD146 & PE-conjugated & BD & 550315 \\
& SSEA4 & PE-conjugated & BD & 560128 \\
& STRO-1 & FITC-conjugated & Biolegend & 340105 \\
Serums & HLA & Rabbit monoclonal & Abcam & 52922 \\
& CD68 & Mouse monoclonal & AbD Serotec & MCA341R \\
& TGF-13I & Rabbit monoclonal & Abcam & 170874 \\
& SOD-1 & Rabbit monoclonal & Abcam & EP1727Y \\
& & & & \\
\cline { 2 - 5 } & Medium and Serum & Source & Catalog No. \\
\cline { 2 - 5 } & KSFM & & Gibco & 17005042 \\
& DMEM & & Gibco & $41966-052$ \\
& & & 26140079
\end{tabular}

\section{Osteogenic and adipogenic differentiation of USC}

\section{Adipogenic induced differentiation}

USC were seeded at a density of 21,000 cells $/ \mathrm{cm}^{2}$ and cultured in serum containing DMEM low-glucose medium with $1 \mu \mathrm{M}$ dexamethasone, $500 \mu \mathrm{M}$ 3-isobutyl-1-methylxanthine, $10 \mu \mathrm{g} / \mathrm{ml}$ insulin, and $100 \mu \mathrm{M}$ indomethacin for 28 days with medium changes every third day. Differentiated cells were then fixed with $4 \%$ paraformaldehyde for $30 \mathrm{~min}$ at room temperature and stained with fresh $0.3 \%$ Oil Red O solution for $50 \mathrm{~min}$. An inverted microscope was used to identify red stained areas, indicating fat droplets. Adipose derived stem cells were used as positive controls.

\section{Osteogenic induced differentiation}

USC were seeded at a density of 4,000 cells $/ \mathrm{cm}^{2}$ and cultured in serum containing DMEM low-glucose medium with $100 \mathrm{nM}$ dexamethasone, $10 \mathrm{mM}$ $\beta$-glycerophosphate and $50 \quad \mu \mathrm{M}$ ascorbic acid-2-phosphate (Wako Chemicals, Richmond, VA) for 28 days with medium changes every third day. Differentiated osteocytes were then fixed by ice-cold $95 \%$ ethanol for 5 minutes at $4{ }^{\circ} \mathrm{C}$ and stained for calcium deposits with $2 \%$ Alizarin Red Solution $(\mathrm{pH}$ 4.0). An inverted microscope was used to identify orange-red stained areas, indicating calcium deposits.

\section{An athymic rat model of dual injury-induced CKD}

Animal experiments were approved by Wake Forest Institutional Animal Care and Use Committee (Protocol no A11-085). Animals were housed in a temperature-controlled environment with free access to food and water. A 12-hour light and 12-hour dark cycle was provided.

To avoid the potential risk of immunoreaction caused by the implanted xenogenic cells, athymic rats (RNU316) were used for this study. These athymic nude rats were T-cell deficient and demonstrated depleted cell populations in thymus-dependent areas of peripheral lymphoid organs. In total, 58 male athymic rats (age 9 weeks, Charles River, NC) were used in this study. To optimize the doses of gentamicin and renal ischemia time frame, 30 animals were used for creation model of dual injury-induced CKD before USC or PBS injection. Among these animals, 22 animals died and 8 animals recovered renal function (failure of model creation) during the model creation processes.

Once the model was successfully established, 28 animals were divided into three groups: a PBS-treated group $(n=9)$, an USC-treated group $(n=9)$, and an age-matched control group $(\mathrm{n}=10)$. A CKD model was induced by creating an ischemia-reperfusion injury and gentamicin injection as demonstrated in Figure $\mathbf{1}$ 28. Briefly, the rats were anesthetized and laid in the supine position with continuous $4 \%$ isoflurane via nose cone. A midline incision was made and intestines were retracted to expose both kidneys. Using vascular clamps, the renal pedicles were clamped bilaterally, for 60 minutes and then released to induce ischemia-reperfusion injury. The Histology clamps were then removed and the analysis incision was closed. Two weeks after surgically-created renal ischemia injury, gentamicin $(100 \mathrm{mg} / \mathrm{kg} /$ day, Phoenix Pharmaceutical Saint Joseph, $\mathrm{MO})$ was injected subcutaneously for 5 consecutive days. Serum creatinine levels were used to evaluate renal function. Blood and urine samples were collected every 2 weeks and

Figure 1. A brief flow chart of the study design. The CKD model was created using dual-injury of renal ischemia and nephrotoxicity using 5 consecutive days of gentamycin injection, 2 weeks after renal ischemia-reperfusion injury. Eight weeks after confirmation of model creation using serial serum $\mathrm{Cr}$ and GFR, animals received either USC or PBS via direct injection into renal parenchyma. Renal function was performed at different time points. Finally, histological and immunocytochemical analysis were performed 12 weeks after injection. Age matched animals were applied as controls (AMC). 
analyzed with blood chemistry machine (Beckman Coulter Inc., Brea, CA). Glomerulus filtration rate (GFR) was calculated as follows: GFR=urine creatinine $\mathrm{x}$ urine volume/serum creatinine/time.

\section{Injections of USC into rat renal parenchyma}

Eight weeks after the dual injury model was created, a USC cell suspension was injected into the kidneys for the USC-treated group. The abdominal contents were exposed once again and both kidneys were identified. A cell suspension of USC was administered into the parenchyma of both kidneys. Each kidney received a total of $5 \times 10^{6} / \mathrm{ml}$ in $100 \mu \mathrm{l}$ of PBS, 8 weeks after CKD creation. To distribute injected cells evenly, $50 \%$ of the aforementioned cell suspension was injected into the upper pole and lower pole of each kidney. PBS $(100 \mu \mathrm{l})$ only was injected in the same fashion as above for the PBS-control group. The model was not created in aged-matched control animals and no injections were performed. Blood and urine samples were collected to test renal function parameters i.e. serum creatinine and GFR, at 2 week intervals. Kidneys were harvested from each animal for histological evaluation, 12 weeks after injection.

\section{Histological and Immuno-histochemical analysis}

Harvested kidney tissues were fixed in 10\% paraformaldehyde solution and processed for paraffin embedding. Five $\mu \mathrm{m}$ sections were obtained. Slides were stained with hematoxylin and eosin (H\&E), and numbers of glomeruli were counted under high power fields (200X) microscope (Leica, Germany). Normal glomeruli are uniform in size, visceral (inner) structures and Bowman's space. Amounts of collagen deposited were assayed by computerized ImageJ (ImageJ software package, NIH) based on Masson's Trichrome staining. Immunohistochemistry was performed with anti-human leukocyte antigen (HLA) and anti CD68 antibodies as a marker of macrophage (BD Biosciences, CA). All slides were blocked in serum-free protein blocker (DAKO, Carpinteria, CA) for 20 minutes.

Transforming growth factor beta 1 (TGF-B1, DAKO) was tested as dysregulation of TGF- $\beta$ activation and signaling may result in apoptosis of resident functional cells but proliferation of fibroblast. In addition, superoxide dismutase 1 (SOD-1) as an antioxidant enzyme protecting the cell from reactive oxygen species toxicity were tested in USC-treated group, compared to PBS-treated control. Antisuperoxide dismutase 1 antibody (EPI722Y) was used at a 1:400 dilution. After incubation with the primary antibodies, tissue sections were incubated with biotin-conjugated secondary antibodies (1:300 dilution) (Vector Laboratories, Burlingame, CA, USA) at room temperature for $60 \mathrm{~min}$, followed by incubation with HRP-conjugated streptavidin (Vector Laboratories) for $30 \mathrm{~min}$ at room temperature and further developed with a DAB substrate kit (Vector Laboratories).

Positive cells were counted under lower and high power fields (100X, 400X). Total number of glomeruli with abnormal structures, increased glomerular size, pathological changes of renal tube, intestinal fibrosis, collagen deposits and inflammatory were quantified. The degree of histological abnormalities on nephrons, such as dilation or collapse, epithelium and obliterated lumen necrosis in renal tubule and glomerular was evaluated. Quantitative comparison of histology and immunohistochemical staining was measured by digital image analysis.

\section{Statistical analysis}

All data were analyzed using software Statistical Package for the Social Sciences (SPSS) version 16.0 for windows (SPSS, Inc., Chicago, IL). Normality of the analyzed data was corroborated with Shapiro-Wilk test. When parametric analysis was possible, data were expressed as mean \pm SD and one-way analysis of variance (ANOVA) was used for comparison among the three groups. Least Significant Difference test was applied to further compare differences among three groups. $\quad \mathrm{P}<0.05$ was considered statistically significant.

\section{Results}

Individual USC appeared consistently as rice grain-like at primary culture (Supplementary Figure 1). USC at passage 3-5 were strong positive for glomerular parietal epithelial cells (CD24, CD29 and CD44), and also classical cell surface markers for MSC (CD73, CD90, CD105, CD146 and SSEA-4), but lack of the expression of hematopoietic markers (CD 31, CD34 and CD45). These results were consistent with our previous reports (Supplementary Figure 1) ${ }^{12-20}$. In addition, USC differentiated into osteogenic and adipogenic cell lineages, respectively (Figure 2).

For evaluation of renal function, serum creatinine $(\mathrm{Cr})$ was evaluated and GFR was calculated every 2 weeks. Creatinine rise and GFR depression were used as indicators of effective CKD model creation. Creatinine levels increased significantly in all animals assigned to USC and PBS groups after one week, post-model creation and remained elevated compared to AMC $(\mathrm{p}<0.01)$. Likewise, GFR declined significantly for animals assigned to USC and PBS 
groups after one week, post-model creation $(\mathrm{p}<0.01)$. The changes in creatinine and GFR were similar in both USC and PBS groups until injection was performed at 13 weeks post-model creation. In the USC-treated group, serum creatinine decreased and GFR increased significantly improved compared to PBS-treated animals two weeks after cell implantation $(p=0.027$ and $p=0.037$, respectively). Th difference in GFR and Cr remained significantly different between these groups until the study endpoint 12 weeks after cell implantation. GFR in the USC-treated group improved to $50 \%$ of that in AMC whereas PBS-treated animals only exhibited a $25 \%$ recovery of GFR compared to AMC (Figure 3).

Grossly, the surface of the kidneys in PBS-treated group was coarse and pale, while kidneys in USC-treated group appeared similar to AMC (Figure 4). In addition, the kidney weight in PBS-treated group $(1.48 \pm 0.31 \mathrm{~g})$ was significantly lower compared to those of the AMC $(1.98 \pm 0.19 \mathrm{~g})$ and USC-treated groups $(1.69 \pm 0.22 \mathrm{~g}) 12$ weeks after injection therapy. The kidney weight in USC-treated group significantly increased compared to that in PBS-treated group $(p<0.01)$ despite not returning to kidney weights exhibited in the AMC group.

Immunocytochemical analysis using HLA staining revealed that the implanted human USC were present around Bowman's capsule or scattered within renal tubules for the entire study period, however numbers of the grafted cell decreased significantly, 12 weeks after injection compared to immediately post-injection (Figure 5). To determine the reno-protective effects of USC, we evaluated the changes in glomerular, renal tubular, and tubulo-interstitial structure among the three groups. In the PBS-treated group, about $38 \%$ of glomeruli displayed normal architecture $(4.0 \pm 2.0$ normal glomeruli/high power field [HPF]) within the renal cortex while $62 \% \quad(6.6 \pm 2.0)$ demonstrated glomerulosclerosis evidenced by wrinkling and collapse of the basement membrane and constricted glomerular capillaries, compared to AMC. This pathological changes in nephrons were significantly lower than the USC-treated group $(6.3 \pm 2.1)$ and the AMC group $(10.6 \pm 2.2) \quad(p<0.01)$. Conversely, the USC-treated group demonstrated $60 \%$ normal glomeruli with $40 \%$ displaying glomerular sclerosis and dilated, atrophic tubules (Figure 6) compared to the AMC group $(\mathrm{p}<0.01)$.

Collagen deposition in the renal parenchyma was investigated using Masson's Trichrome staining (Figure 7). Collagen deposition was elevated in PBS-treated animals (28.4 \pm 6.3$)$. This was significantly higher than in USC-treated group $(8.3 \pm 3.0)$ and the AMC group $(1.3 \pm 0.3) \quad(\mathrm{p}<0.01)$, which represented over a 20 -fold increase. There was also significantly higher collagen deposition in the USC-treated group compared to the AMC group which was only a 6-fold increase. To assess monocyte infiltration (Figure 8), numbers of cells expressing $\mathrm{CD}^{+} 8^{+}$was significantly higher in the PBS group $(8.7 \pm 2.54)$ compared to both USC-treated group $(4.7 \pm 2.12)$ and AMC group $(2.0 \pm 1.05)(\mathrm{p}<0.01)$. The USC-treated group exhibited an elevated numbers of cells expressing CD68 ${ }^{+}$compared to the AMC group as well, however albeit not as drastic.

To evaluate tissue fibrosis within the renal tissues, TGF- $\$ 1$ as an inflammatory marker (Figure 9) was used. TGF-131 expression was significantly high in the PBS-treated groups in both the cortex and medulla $(1.2 \pm 0.1$ and $1.3 \pm 0.08)$ which represented a $58 \%$ and $69 \%$ increase compared to AMC $(0.8 \pm 0.05$ and $0.7 \pm 0.06)$. Expression of TGF- $B 1$ in the cortex and medulla of the USC-treated group $(0.95 \pm 0.09$ and $0.99 \pm 0.06)$, respectively, represented as $23 \%$ and $29 \%$ increase compared to AMC. The relative increase in TGF-B1 for PBS-treated animals was significantly higher than for the USC-treated group $(\mathrm{p}<0.01)$.
A

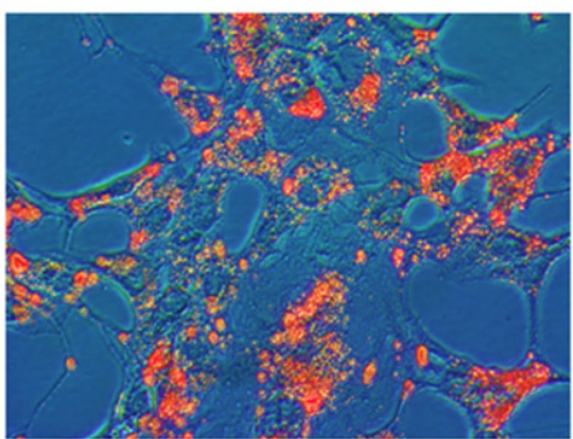

Adipogenic differentiation of USC

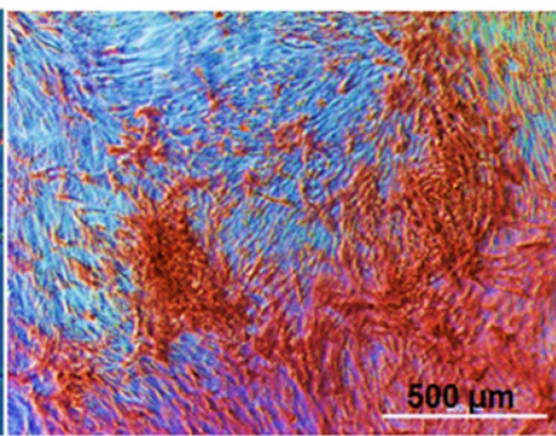

Osteogenic differentiation of USC

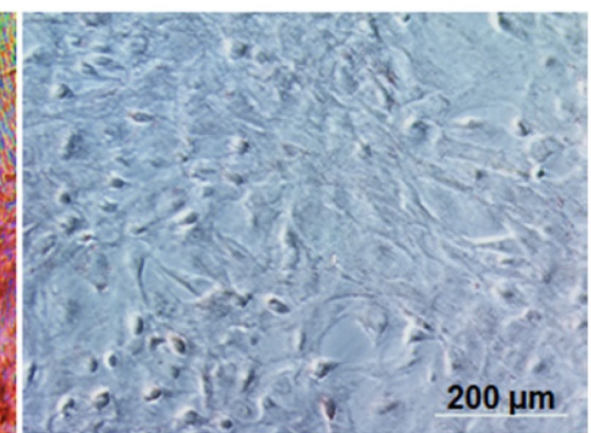

Non-induced USC

Figure 2. In vitro differentiation potential of USC. (A) Adiopogenic differentiation of USC was demonstrated with oil red O (lipid droplet). (B) Osteogenic differentiation of USC was demonstrated with Alizarin Red. (C) Non-induced USC acted as a control group. 
required. Herein, we have established a model using ischemia-reperfusion injury combined with gentamicin-induced nephrotoxicity to provide an easy and reliable animal model to study CKD 28 .

Given that definitive treatment i.e. renal transplant is not commonplace; it is of utmost importance to prevent progression of CKD to ESRD. The nephron is the functional unit of the kidney and thus we quantified the number of normal nephrons in each treatment group. In the USC treated group, 70\% of glomeruli displayed normal architecture while this number decreased to $40 \%$ in the PBS-treated group. In contrast, numbers of abnormal nephrons significantly lower in USC-treated group than those in PBS group. Likewise there was a significant decrease in serum $\mathrm{Cr}$ and increase in GFR in the USC group compared to PBS treated animals. It is likely that the preservation of glomerular architecture, evidenced by a lower level of glomerulosclerosis and dilatated, atrophic tubules, in the USC treated group compared to the PBS group is responsible for the chemical (serum $\mathrm{Cr}$ ) and functional (GFR) findings.
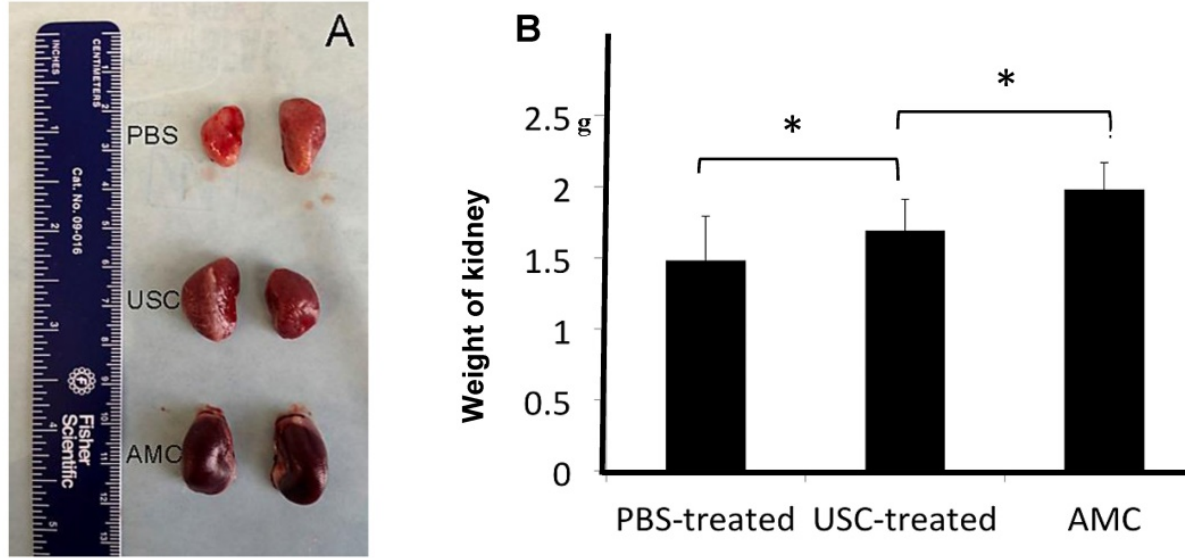

Figure 4. The gross appearance and weight changes of the kidneys. (A) The gross appearance of kidneys in the three treatment groups was noted. (B) The weight of the kidneys in USC-treated rats was significantly elevated compared to the PBS-treated animals but also significantly lighter than that in AMC group, $*$ Significance at the $p<0.05$ level.
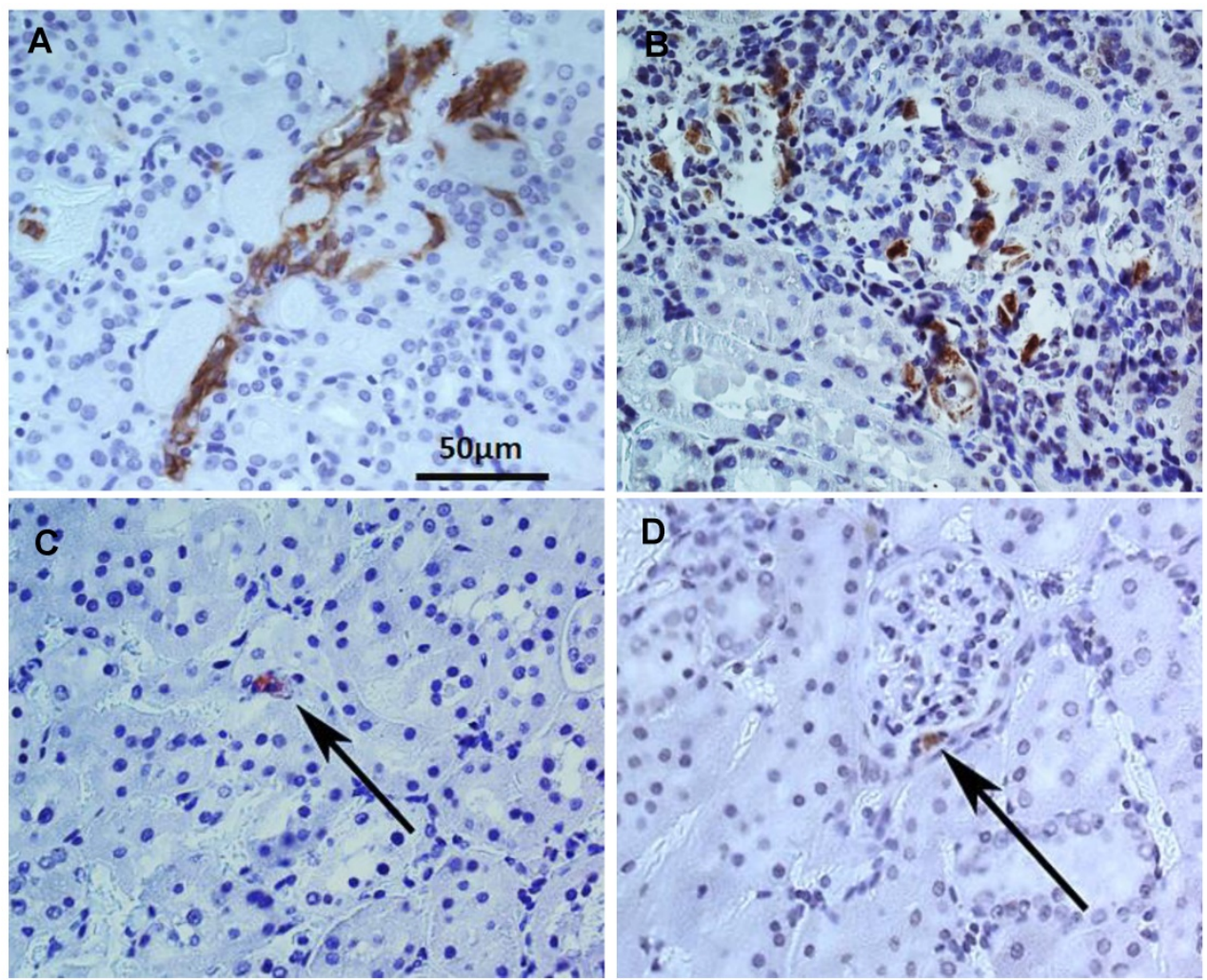

Figure 5. Tracking implanted USC within renal tissues by immuncytochemistry analysis with anti-HLA A antibody. More grafted USC (brown) were found in the tubule interstitial regions one week $(\mathbf{A})$ and two weeks (B) after implantation compared to 12 weeks. Number of the implanted USC decrease with time during the 12 week follow-up. A few cells were still identified in the renal tubules and the medulla (C) as well as around Bowman's capsule in the renal cortex (D). 

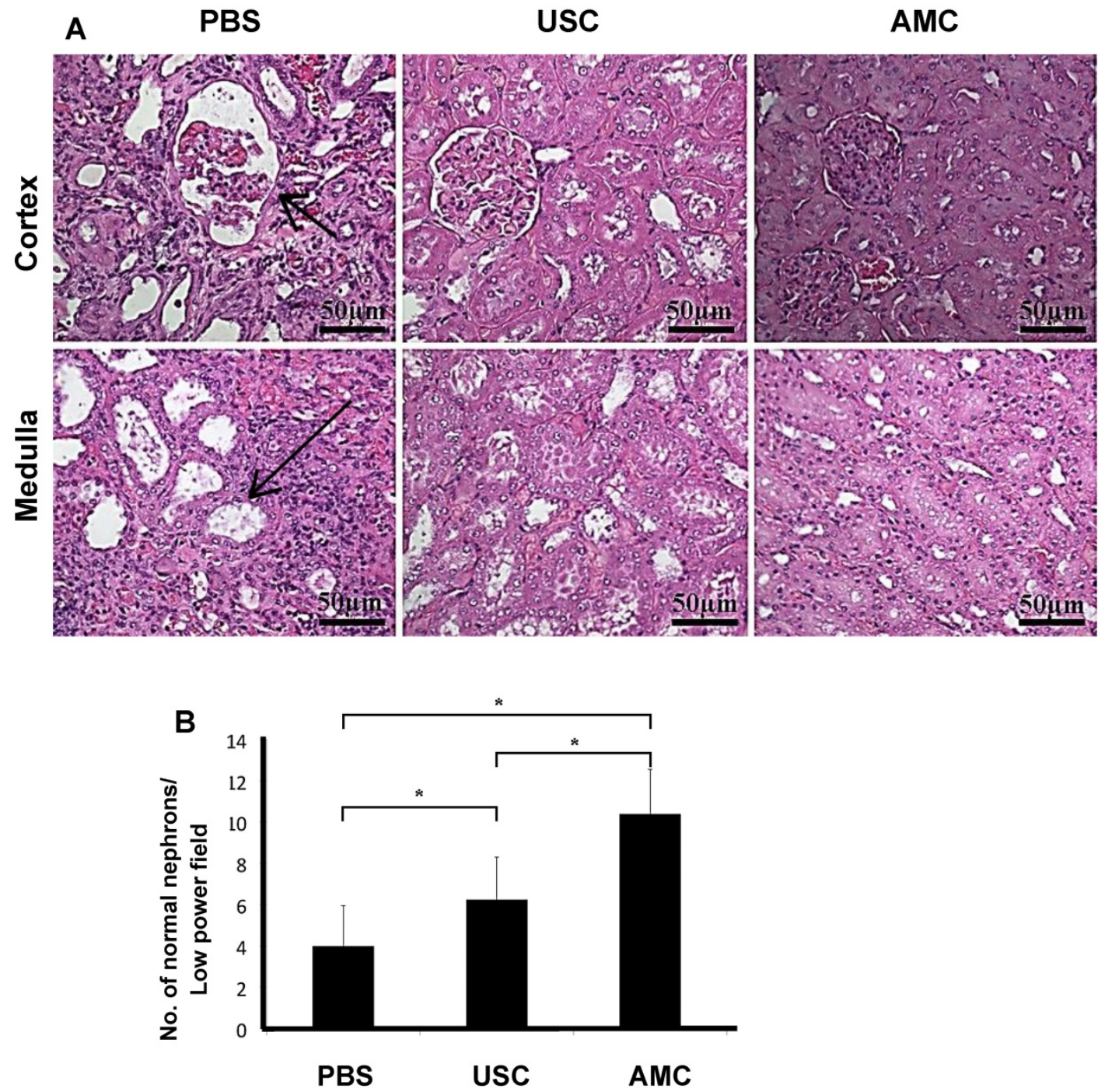

Figure 6. of Patho-histological changes in nephrons within renal cortex and medulla after USC implantation. (A) About $60 \%$ of glomeruli increased in size with collapse of some glomerular tufts (short arrow) within the renal cortex and $62 \%$ of renal tubules were dilated (long arrow) within the medulla in PBC-treated rats. In contrast, a majority of glomeruli and renal tubules displayed normal structure and only $40 \%$ of nephrons displayed abnormal configuration in USC-treated group. (B) Expressed as the average numbers of relative abnormal nephrons in six fields per sample at 200x magnification. Numbers of normal nephron within renal tissue under high power field with H\&E staining. * Significance at the $p<0.05$ level.

Tissue fibrosis and collagen deposition are important contributors to progressive renal failure. This is a complex process involving fibroblasts, immune reactions and free radical damage to the renal parenchyma. We evaluated these processes through gross examination of the kidneys and through the chemical investigation of mediators of fibrosis and free radical damage. Monocyte infiltration as cells expressing $\mathrm{CD}^{+} 8^{+}$was elevated in the PBS group to a greater extent than in USC treated group, compared to AMC. TGF- $B 1$ as a surrogate for tissue fibrosis followed a similar trend. Superoxide dismutase is an important mediator in inhibiting free radical induced tissue damage and its expression was lowest in the PBS treated group and highest in the USC treated group. Given the lower levels of TGF- $B 1$ and $\mathrm{CD}_{68}{ }^{+}$cells in the USC group compared to the
PBS group, it is unsurprising the see a lower level of collagen deposition and improved renal function in the latter (Figure 9). Furthermore, collagen and fibrosis lead to glomerular and tubular atrophy, explaining the gross appearance and the weights of the kidneys in the PBS group compared to the USC and AMC groups.

Our study is not without limitations which may need to be addressed prior to beginning safety trials. First, we only administered a single dose of a given concentration of USC. It is feasible that multiple injections may further improve the renoprotection imbued by USC. These injections may need to be distributed over several weeks to months for maximal effect. Second, it is possible that increasing the number of injected cells will improve kidney function even further. 

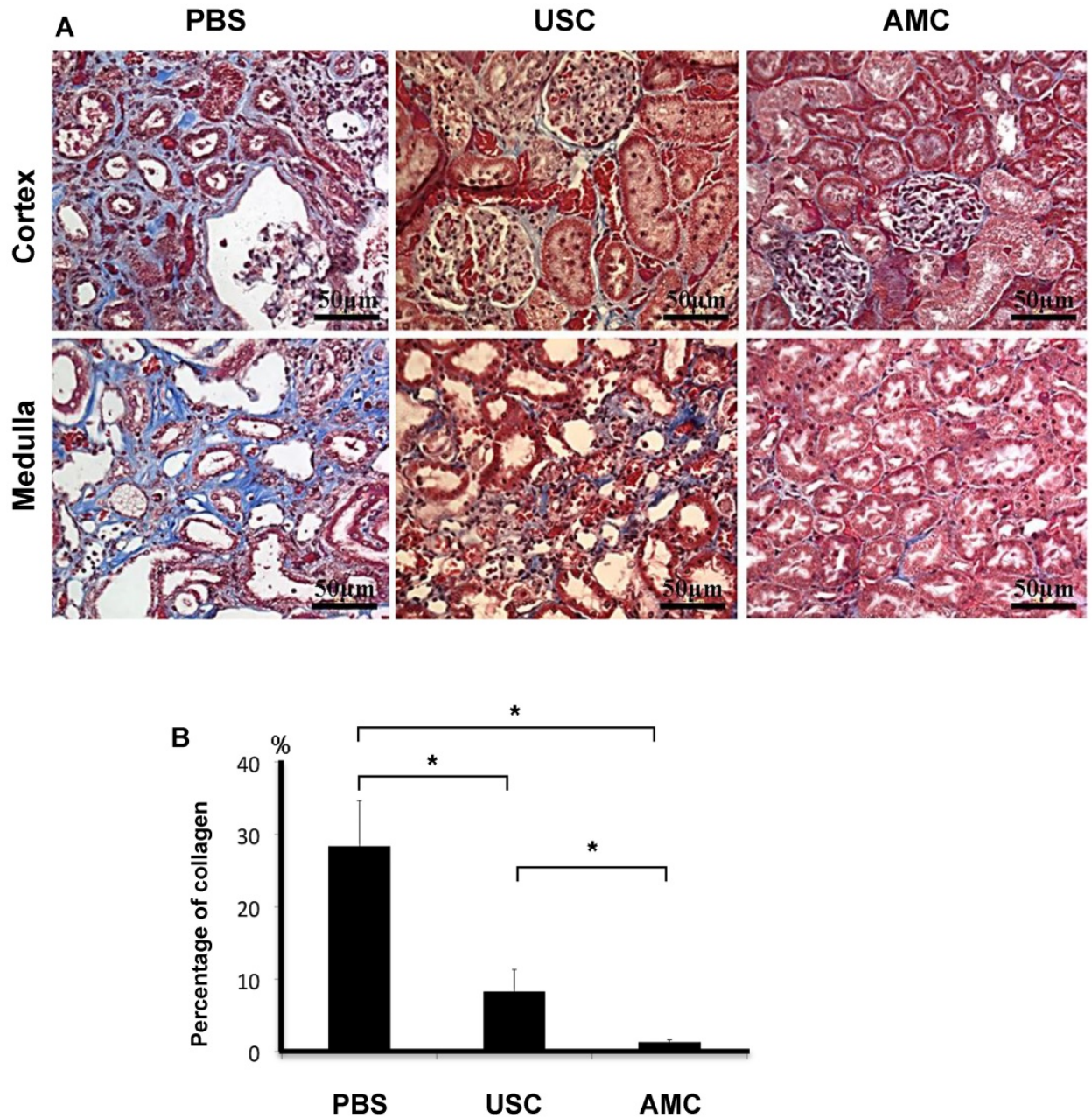

Figure 7. Histological appearance of collagen deposition and fibrotic changes of the kidneys. (A) Among of collagen deposition in Masson Trichrome staining was significantly elevated in PBS-treated rats, and minimal changes in kidney of rats treated with USC-treated group compared to that in AMC. (B) Percentage of collagen deposition within the renal parenchyma. * Significance at the $p<0.05$ level
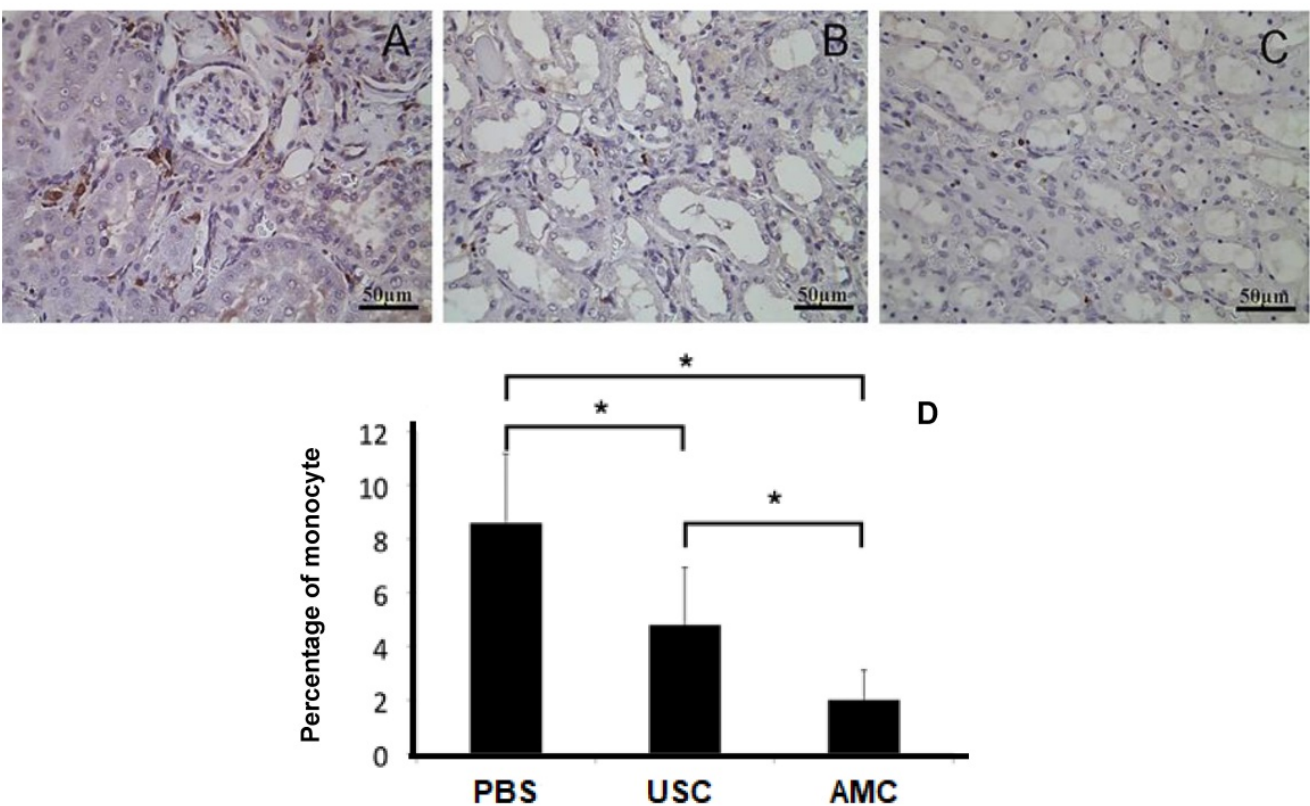

Figure 8. CD68 expression as a surrogate for monocyte infiltration within the kidney. (A) Number of infiltrated monocyte expressing positive for CD68 significantly increased in PBS-treated group, compared to AMC. (B) Number of cells expressing CD68 significantly decreased in USC group compared (C) compared to PBS group. (D) Analysis of CD68 expression in the three groups with immunocytochemical staining. * Significance at the $p<0.05$ level 
TGF $\beta$-1 expression in renal tissues
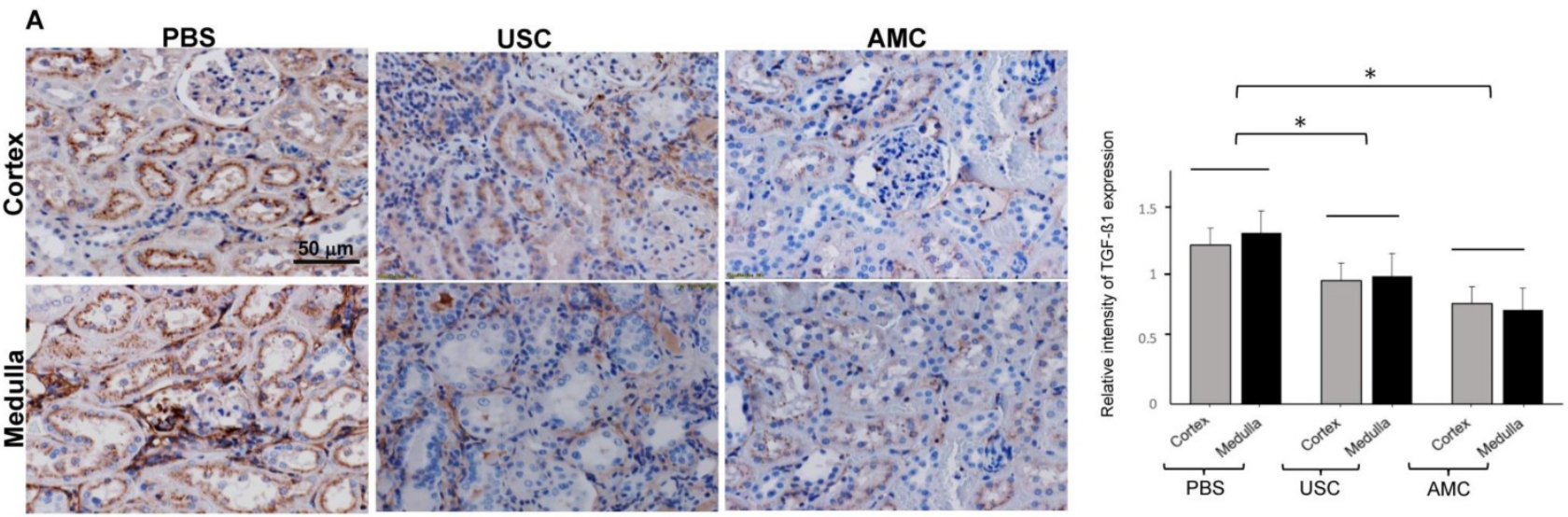

\section{SOD-1 expression in renal tissues}
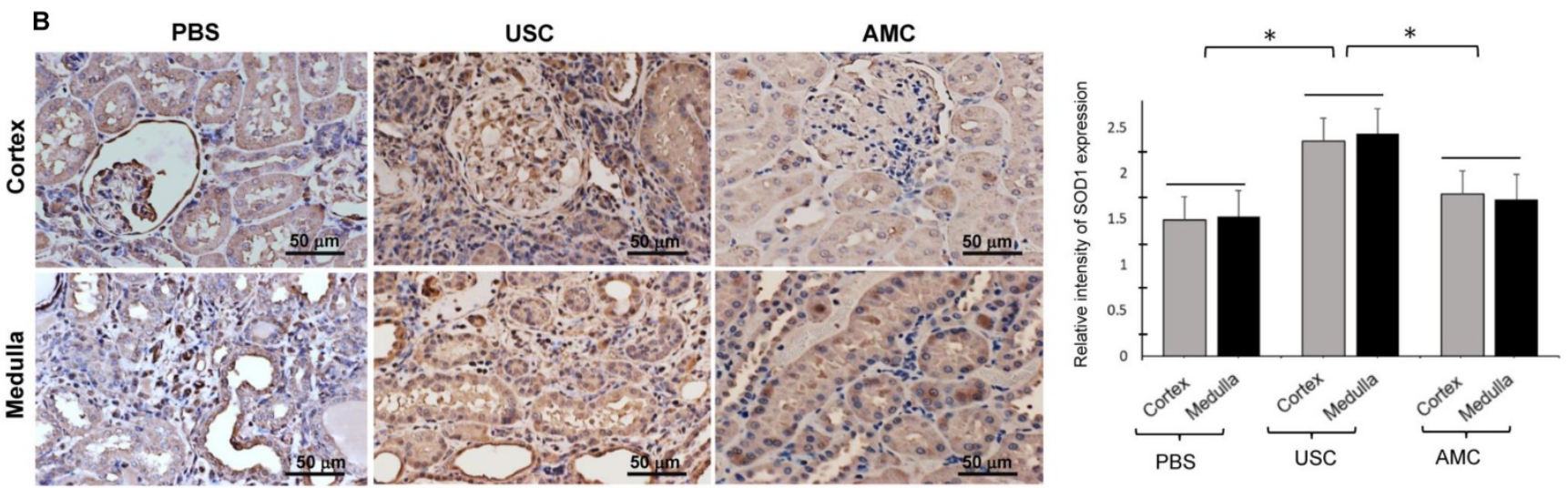

Figure 9. Effect of USC implantation on anti-fibroblast and redox properties in CKD rodent model. (A) Amount of TGF- $\beta 1$ expression significantly increased in renal cortex and medullar tissue of PBS treated animals compared to that of AMC group. However, USC implantation significantly decreased levels of TGF- $\beta 1$ expression, compared PBS treatment. (B) Amount of SOD-1 expression levels significantly increased in renal tissue of USC-treated groups compared to that of PBS group. Semi-quantification is expressed as the average relative inte nsity of six fields around defect site per sample, each taken at a $200 x$ magnification. Representative images are shown at $200 x$ magnification. ${ }^{*} p<0.01$ compared to $A M C$ as a unit set.

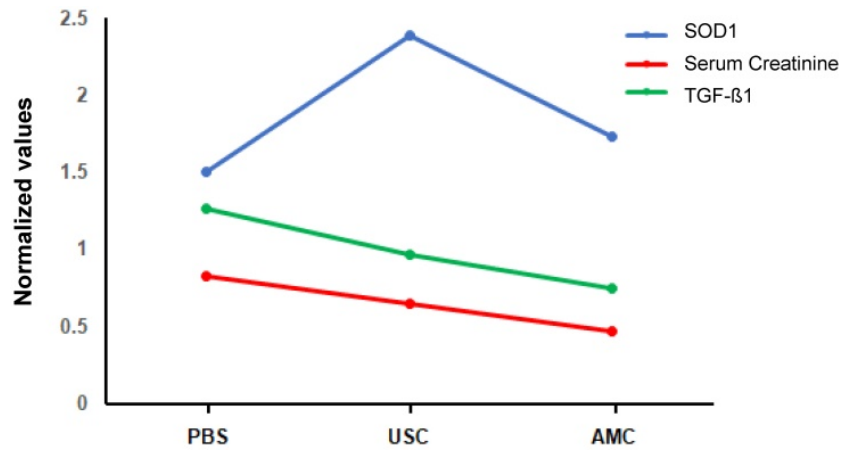

Figure 10. Relationship between serum creatinine and both the oxidative stress parameter and the inflammatory parameters with regression line analysis. Antioxidant and antiinflammatory activity of USC is behind the renoprotective activity, compared to those of PBS-treated and AMC groups.

Third, very few studies have been conducted investigating routes of administration for urologic stem cell therapy and thus it may be that intravenous therapy may provide improved benefit over intrarenal injection. Finally, molecular analysis of pro-regenerative, anti-apoptotic or anti-fibrotic pathways needs further investigation.

Herein, we have demonstrated that local implantation of human USC significantly improved renal function, imbued a protective effect on nephrons, reduced renal scaring, and mollified the inflammatory response in a renal ischemiareperfusion, gentamicin, dual injury-induced CKD rodent model (Figure 10). Through our chemical and functional analysis, it is clear that USC have some impact on the aforementioned parameters insofar as USC treatment ameliorates renal degradation seen in the group that received no treatment.

\section{Abbreviations}

AMC: age-matched control; CKD: chronic kidney disease; ESRD: end-stage renal disease; H\&E: hematoxylin and eosin; HLA: human leukocyte antigen; HPF: high power field; FITC: Fluorescein isothiocyanate; GFR: Glomerulus filtration rate; iPSC: induced pluripotent stem cells; PBS: phosphate 
buffered saline; PE: phycoerythrin; SSEA-4: stagespecific embryonic antigen-4; SPSS: Statistical Package for the Social Sciences; USC: Urine-derived stem cells.

\section{Supplementary Material}

Supplementary figures and tables.

http://www.ijbs.com/v16p0435s1.pdf

\section{Acknowledgements}

The authors would like to acknowledge all parties that participated in this study. Authors also would like to thank Dr. Marshall Z. Schwartz in Department of Urology at Wake Forest School of Medicine for his editing and helpful discussions.

\section{Author contributions}

CZ, SG, MA, TK, and PT carried out the cell culture, molecular studies, the animal experiments and drafted the manuscript. CZ, SG, and PT carried out immunoassays and performed statistical analyses. $\mathrm{AA}$ and $\mathrm{YZ}$ participated in the design of the study. YZ, IK, and PT conceived the study, participated in its design, and completed the manuscript. JY, YS, JJ and AA participated in administration and coordination. All authors read and approved the final manuscript.

\section{Ethics approval and consent to participate}

Collection of human urine samples in this study was approved by the Wake Forest University Health Sciences Institutional Review Board and was performed in accordance with the Declaration of Helsinki (2004). Signed, informed consent was collected from patients.

\section{Ethics approval for animal study}

Rodent experiments were approved by Wake Forest Institutional Animal Care and Use Committee (IACUC).

\section{Competing Interests}

The authors have declared that no competing interest exists.

\section{References}

1. Centers for Disease Control and Prevention. National Chronic Kidney Disease Fact Sheet, 2017.

2. Awdishu L, Mehta RL. The 6R's of drug induced nephrotoxicity. BMC Nephrol 2017;18:124.

3. Vanholder R, Lameire N, Annemans L, Van Biesen W. Cost of renal replacement: how to help as many as possible while keeping expenses reasonable? Nephrology Dialysis Transplantation 2015;31:1251-61.

4. Hickson LJ, Eirin A, Lerman LO. Challenges and opportunities for stem cell therapy in patients with chronic kidney disease. Kidney Int 2016;89:767-78

5. Prodromidi EI, Poulsom R, Jeffery R, et al. Bone marrow-derived cells contribute to podocyte regeneration and amelioration of renal disease in a mouse model of Alport syndrome. Stem cells 2006;24:2448-55.

6. Noronha IL, Cavaglieri RC, Janz FL, et al. The potential use of stem cells derived from human amniotic fluid in renal diseases. Kidney international supplements 2011;1:77-82.
7. Rota C, Imberti B, Pozzobon M, et al. Human amniotic fluid stem cell preconditioning improves their regenerative potential. Stem cells and development 2012;21:1911-23.

8. Sangidorj $\mathrm{O}$, Yang SH, Jang HR, et al. Bone marrow-derived endothelial progenitor cells confer renal protection in a murine chronic renal failure model. American journal of physiology Renal physiology 2010;299:F325-35.

9. Yokote S, Katsuoka Y, Yamada A, Ohkido I, Yokoo T. Effect of adipose-derived mesenchymal stem cell transplantation on vascular calcification in rats with adenine-induced kidney disease. Scientific reports 2017;7:14036.

10. Yamaleyeva LM, Guimaraes-Souza NK, Krane LS, et al. Cell therapy with human renal cell cultures containing erythropoietin-positive cells improves chronic kidney injury. Stem Cells Transl Med 2012;1:373-83.

11. Makhlough A, Shekarchian S, Moghadasali R, et al. Bone marrow-mesenchymal stromal cell infusion in patients with chronic kidney disease: A safety study with 18 months of follow-up. Cytotherapy 2018;20:660-9.

12. Zhang $\mathrm{Y}, \mathrm{McNeill} \mathrm{E}$, Tian $\mathrm{H}$, et al. Urine derived cells are a potential source for urological tissue reconstruction. J Urol 2008;180:2226-33.

13. Bharadwaj S, Liu G, Shi Y, et al. Characterization of urine-derived stem cells obtained from upper urinary tract for use in cell-based urological tissue engineering. Tissue engineering Part A 2011;17:2123-32.

14. Bharadwaj S, Liu G, Shi Y, et al. Multipotential differentiation of human urine-derived stem cells: potential for therapeutic applications in urology. Stem cells 2013;31:1840-56.

15. Lang R, Liu G, Shi Y, et al. Self-renewal and differentiation capacity of urine-derived stem cells after urine preservation for 24 hours. PloS one 2013;8:e53980.

16. Liu G, Pareta RA, Wu R, et al. Skeletal myogenic differentiation of urine-derived stem cells and angiogenesis using microbeads loaded with growth factors. Biomaterials 2013;34:1311-26.

17. Liu G, Wu R, Shi Y, Deng C, Zhou X, Atala A, Opara E, Zhang Y. Skeletal Myogenic Differentiation of Urine-Derived Stem Cells, Angiogenesis and Innervation Using Hydrogel Loaded with Growth Factors for Potential in Treatment of Urinary Incontinence. J Urol. 2015;193:e74.

18. Liu Y, Ma W, Liu B, et al. Urethral reconstruction with autologous urine-derived stem cells seeded in three-dimensional porous small intestinal submucosa in a rabbit model. Stem cell research \& therapy 2017;8:63.

19. Wu RP, Liu G, Shi YA, Bharadwaj S, Atala A, Zhang Y. Human urine-derived stem cells originate from parietal stem cells. J Urol 2014;191:e1-e958.

20. Wu S, Liu Y, Bharadwaj S, Atala A, Zhang Y. Human urine-derived stem cells seeded in a modified $3 \mathrm{D}$ porous small intestinal submucosa scaffold for urethral tissue engineering. Biomaterials 2011;32:1317-26.

21. Zhang D, Wei G, Li P, Zhou X, Zhang Y. Urine-derived stem cells: A novel and versatile progenitor source for cell-based therapy and regenerative medicine. Genes Dis 2014;1:8-17.

22. Qin D, Long T, Deng J, Zhang Y. Urine-derived stem cells for potential use in bladder repair. Stem Cell Res Ther 2014;5:69.

23. Long TJ, et al. Human urine-derived stem cells lessen inflammation and fibrosis within kidney tissue in a rodent model of aging-related renal insufficiency. J Urol 2017;197:e293.

24. Dong X, Zhang T, Liu Q, et al. Beneficial effects of urine-derived stem cells on fibrosis and apoptosis of myocardial, glomerular and bladder cells. Molecular and cellular endocrinology 2016;427:21-32.

25. Jiang ZZ, Liu YM, Niu X, et al. Exosomes secreted by human urine-derived stem cells could prevent kidney complications from type I diabetes in rats. Stem Cell Res Ther 2016;7:24.

26. Tian SF, Jiang ZZ, Liu YM, et al. Human urine-derived stem cells contribute to the repair of ischemic acute kidney injury in rats. Molecular medicine reports 2017;16:5541-8.

27. Sagrinati C, Netti GS, Mazzinghi B, et al. Isolation and characterization of multipotent progenitor cells from the Bowman's capsule of adult human kidneys. Journal of the American Society of Nephrology : JASN 2006;17:2443-56.

28. Wang HJ, Varner A, AbouShwareb T, Atala A, Yoo JJ. Ischemia/reperfusion-induced renal failure in rats as a model for evaluating cell therapies. Ren Fail 2012;34:1324-32.

29. Ozbek E, Cekmen M, Ilbey YO, Simsek A, Polat EC, Somay A. Atorvastatin prevents gentamicin-induced renal damage in rats through the inhibition of p38-MAPK and NF-kappaB pathways. Ren Fail 2009;31:382-92.

30. Basile DP, Anderson MD, Sutton TA. Pathophysiology of acute kidney injury. Compr Physiol 2012;2:1303-53.

31. Yang HC, Zuo Y, Fogo AB. Models of chronic kidney disease. Drug Discov Today Dis Models 2010;7:13-9. 
32. Kelley R, Werdin ES, Bruce AT, et al. Tubular cell-enriched subpopulation of primary renal cells improves survival and augments kidney function in rodent model of chronic kidney disease. American journal of physiology Renal physiology 2010;299:F1026-39.

33. Caldas HC, Fernandes IM, Kawasaki-Oyama RS, et al. Effect of stem cells seeded onto biomaterial on the progression of experimental chronic kidney disease. Exp Biol Med (Maywood) 2011;236:746-54.

34. Becker GJ, Hewitson TD. Animal models of chronic kidney disease: useful but not perfect. Nephrol Dial Transplant 2013;28:2432-8. 